\title{
ERESBIL-Archivo Vasco de la Música (Errenteria): Una institución para la preservación y difusión de la música
}

\author{
ERESBIL-Archivo Vasco de la Música (Errenteria): \\ An Institution for preservation and diffusion of Music
}

Jon Bagüés

ERESBIL-Archivo Vasco de la Música jbagues@eresbil.com

\section{RESUMEN}

Ofrecemos una presentación de la institución documental ERESBIL, creada en la localidad guipuzcoana de Errenteria en 1974 con el objeto de la investigación, recopilación, conservación y difusión del patrimonio musical y, en especial, la producción de los compositores vascos. A lo largo de las próximas páginas se repasan los contenidos de las colecciones integradas en las distintas secciones, entre las que destacan 180 fondos y colecciones; los catálogos, guías e inventarios disponibles; las actividades de difusión e investigación, así como la cooperación con otras instituciones musicales y documentales.

Palabras clave: Documentación musical, Archivos musicales, Patrimonio musical, País Vasco.

\begin{abstract}
This is an introduction to ERESBIL, a documental institution born in the city of Errenteria (Guipúzcoa) in 1974 with the aim of researching, compiling, preserving and disseminating musical heritage mainly created by Basque composers. In this paper, we are going to review the content of every collection in the different sections, including 180 collections, catalogues, guides and available inventories, activities of dissemination and research, as well as collaborative relationships with other musical and documental institutions.
\end{abstract}

Key words: Musical Documentation, Musical Archives, Musical Heritage, Basque Country. 


\section{ERESBIL-ARCHIVO VASCO DE LA MÚSICA (ERRENTERIA):}

\section{UNA INSTITUCIÓN PARA LA PRESERVACIÓN Y DIFUSIÓN DE LA MÚSICA}

Bagüés, J. (2016). ERESBIL-Archivo Vasco de la Música (Errenteria): Una institución para la preservación y difusión de la música. Cuadernos de Investigación Musical, 1, 60-77.

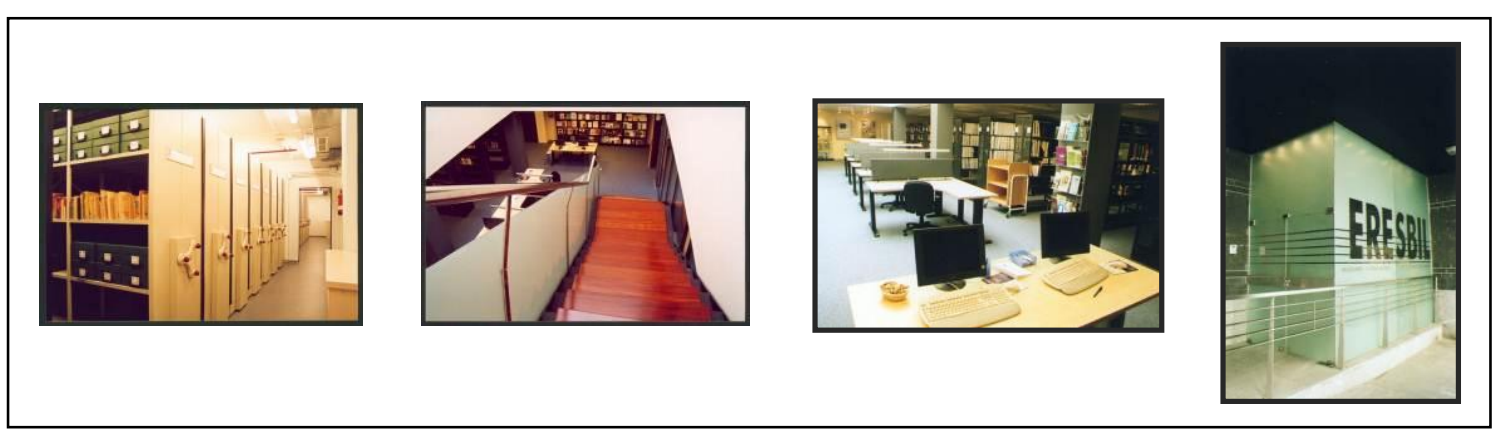

Fig. 1. Creación y evolución de ERESBIL.

\section{INTRODUCCIÓN}

ERESBIL - Archivo Vasco de la música tiene por objeto, según indican los estatutos vigentes (2011) como Fundación, la investigación, recopilación, conservación y difusión del Patrimonio Musical y, en especial, la producción de los Compositores Vascos ${ }^{1}$. Radica en la localidad guipuzcoana de Errenteria, donde nace en 1974 de la idea de José Luis Ansorena, director de la Coral Andra Mari de la citada localidad. Surge con el principal objetivo de servir a la semana musical MUSIKASTE, creada en 1973 para difundir la música de los compositores vascos.

Quizás convenga indicar brevemente un par de circunstancias históricas en relación al desarrollo de la música impresa y su conservación en el País Vasco. Si la evolución de la imprenta musical en España ha sido modesta hasta el siglo XIX, podemos decir que es inexistente en el País Vasco hasta el segundo tercio del siglo XIX. Y aun posteriormente la cantidad y géneros de la música que se edita tiene relación con las posibilidades de venta. $\mathrm{Al}$ margen de algunos autores de la música coral que se han constituido en autoeditores, son pocos los autores que consiguen tener una empresa editora para difundir su creación. Ello significa que la mayor parte de la producción de los compositores en el País Vasco permanece en la copia manuscrita (hasta hace poco en papel, hoy en día en papel y en ordenador).

Por otra parte hay que recordar que ha sido tradición en Europa que las Bibliotecas Nacionales fueran las responsables del patrimonio documental impreso, ocupándose a

\footnotetext{
${ }^{1}$ En la página web de Eresbil, http://www.eresbil.com/ pueden consultarse otros datos de su evolución, secciones así como memorias anuales. Los enlaces a las distintas secciones y páginas web dependientes del sitio web ERESBIL se incluirán como hiperenlaces en el texto. Todos ellos están consultables a diciembre de 2016. Los enlaces a otros sitios ajenos a ERESBIL se indicarán asimismo en nota a pie de página.
} 
JON BAGÜÉS

partir del siglo XIX también de las partituras, a través de secciones de música. Con el desarrollo de las autonomías en España, debemos constatar que el País Vasco es hoy una de las escasas Comunidades Autónomas que no cuenta aún con una Biblioteca General dedicada a la recopilación, custodia y difusión de su patrimonio documental impreso.

Esta puede ser una breve cronología con las fechas más significativas en la evolución de ERESBIL:

. 1973. Se crea en Errenteria (Gipuzkoa) la Semana musical MUSIKASTE.

- 1974. José Luis Ansorena crea ERESBIL-Archivo de Compositores Vascos, apoyado por el Ayuntamiento de Errenteria.

. 1977. Se crea el Patronato Provincial, con representación de la Coral Andra Mari, el Ayuntamiento de Errenteria y la Diputación Provincial de Gipuzkoa.

- 1979. Se inaugura la sede del archivo en los locales de la Fraternidad de Capuchinos de Errenteria, con la asistencia de Manuel Lekuona y Miguel Querol.

. 1986. Se constituye el Patronato ERESBIL, con la presidencia del Gobierno Vasco, formada conjuntamente con la Diputación Foral de Gipuzkoa, el Ayuntamiento de Errenteria, la Comunidad de Capuchinos y la Coral Andra Mari.

. 2000. ERESBIL adopta como expresión de subtítulo el de Archivo Vasco de la Música. Preserva desde este año una copia del Depósito Legal en materia de partituras y registros sonoros de la Comunidad Autónoma Vasca.

. 2002. Se inaugura la sede actual del archivo en la calle Alfonso XI de Errenteria, un edificio de cuatro plantas con una superficie útil de 1.300 metros cuadrados.

ERESBIL es acrónimo de Euskal Ereslarien Bilduma, o Archivo de Compositores Vascos, la inicial orientación en cuanto a sus contenidos. En el año 2000 cambia su denominación por Archivo Vasco de la Música, en gran parte por la evolución de los propios contenidos de la colección documental como iremos explicando a continuación.

\section{CONTENIDO DE LAS COLECCIONES DE ERESBIL}

ERESBIL se articula actualmente en diversas secciones, siendo la inicial la colección relacionada con las partituras de los compositores vasco-navarros de todos los tiempos. Su recolección fue la finalidad inicial del archivo, siendo aún el eje central del mismo.

\subsection{ERESBIL - SECCIÓN DE COMPOSITORES VASCOS}

Inicialmente se centró la labor en conseguir copias, tanto impresas como por otros procedimientos, de la labor creativa de los compositores vascos. Con el tiempo, la relación con las editoriales de partituras, en buena parte de compositores autoeditores, así como la copia que ingresa por Depósito Legal desde el año 2000, se convierte en una fuente de 
ERESBIL-ARCHIVo VASCO DE LA Música (ERRENTERIA):

UNA INSTITUCIÓN PARA LA PRESERVACIÓN Y DIFUSIÓN DE LA MÚSICA

alimentación de esta sección. Desde su creación en 1998 ERESBIL es la sede social de la asociación MUSIKAGILEAK, que agrupa a compositores del País Vasco a ambos lados de la frontera y de Navarra. Gracias a esta relación dispone ERESBIL de una comunicación fluida con la creación musical contemporánea, contando con su asesoría tanto para la semana MUSIKASTE como para la realización de otras actividades.

A fecha de diciembre de 2016 esta sección contiene 33.510 partituras de autores vasco-navarros, con la siguiente especificación de géneros:

$\begin{array}{lr}\text { Canto solista } & 4.312 \\ \text { Coral a capella } & 8.206 \\ \text { Coral con acompañamiento } & 7.717 \\ \text { Piano y clave } & 2.762 \\ \text { Órgano } & 1.136 \\ \text { Acordeón } & 486 \\ \text { Percusión } & 56 \\ \text { Guitarra, arpa } & 409 \\ \text { Instrumentos de viento } & 246 \\ \text { Txistu } & 3.245 \\ \text { Instrumentos de cuerda } & 322 \\ \text { Cámara-viento } & 192 \\ \text { Cámara-cuerda } & 313 \\ \text { Cámara varia sin piano } & 306 \\ \text { Cámara varia con piano } & 709 \\ \text { Instrumentos electrónicos } & 33 \\ \text { Formaciones diversas } & 178 \\ \text { Música para orquesta } & 822 \\ \text { Música sinfónico-coral } & 703 \\ \text { Banda } & 1.075 \\ \text { Música dramática } & 282\end{array}$

TOTAL $\quad 33.510$

\subsection{SECCIÓN DE FONDOS DE ARCHIVO}

ERESBIL recopila desde sus inicios fondos personales e institucionales. Si bien inicialmente eran los compositores y sus obras el principal objeto de búsqueda, con el tiempo se ha ampliado el radio de acción a los intérpretes, instituciones musicales 
JON BAGÜÉS

desaparecidas, o colecciones, tanto familiares como personales. Son más de 180 los fondos que contiene, entre los que podríamos destacar los siguientes:

\section{COMPOSITORES:}

- Valentín Zubiaurre (1837-1914, maestro de la Real Capilla en Madrid)

- Beltrán Pagola (1878-1950, docente en San Sebastián)

. Tomás Mújica (1883-1963, docente en Montevideo, Uruguay)

- Jesús Guridi (1886-1961, director y catedrático de órgano del RCSM de Madrid)

- José Ma Usandizaga (1887-1915)

- José Uruñuela (1891-1963, maestro de danza en San Sebastián)

- Norberto Almandoz (1893-1970, maestro de capilla y director del conservatorio de Sevilla)

- Jesús Arámbarri (1902-1960, director de orquesta y banda en Madrid)

. Luis de Aramburu (1905-1999, organista y docente en Vitoria)

. Rodrigo A. de Santiago (1907-1985, director de orquesta y banda en A Coruña y Madrid)

- Francisco Escudero (1912-2002, docente y director de orquesta)

. Gotzon Aulestia (1940-2003, docente en San Sebastián)

INTÉRPRETES:

- Faustino Sarasola (1873-1929, organista en Régil, Guipúzcoa)

- Gelasio Arámburu (1896-1968, director de coro en Pasajes, Biarritz y San Sebastián)

. Nicanor Zabaleta (1907-1993, arpista)

- Gabriel Verkos (1910-1981, violonchelista en Bilbao)

- José Mendizábal (1920-1998, txistulari en Rentería y Oiartzun)

CRÍTICOS, MUSICÓLOGOS, GESTORES Y PEDAGOGOS

. Ángel Inaraja (c.1908-1982)

- José Ma Zapirain (1914-2007)

. José Antonio Arana-Martija (1931-2011)

\section{INSTITUCIONES}

· Coro Maitea (1945-1977, San Sebastián)

. Escolanía Felipe Gorriti (Tolosa)

- Orfeón de la Prisión Central de Astorga 
ERESBIL-ARCHIVo VASCO DE LA Música (ERRENTERIA):

UNA INSTITUCIÓN PARA LA PRESERVACIÓN Y DIFUSIÓN DE LA MÚSICA

FONOTECAS DE RADIO

. $\underline{\text { Radio San Sebastián (Cadena SER) }}$

.

. Euskadi Irratia (Fonoteca clásica)

- Herri Irratia (San Sebastián)

Recientemente ERESBIL ha publicado online la Guía de Fondos y Colecciones documentales donde además de las mencionadas pueden consultarse los distintos fondos tanto por índice alfabético, como por tipos de fondo o clasificación temática.

De manera global podríamos resaltar una importante presencia de documentación relacionada con la música religiosa y la música coral de finales del s. XIX y s. XX; de la música para piano, para órgano o para guitarra así como de la música para banda.

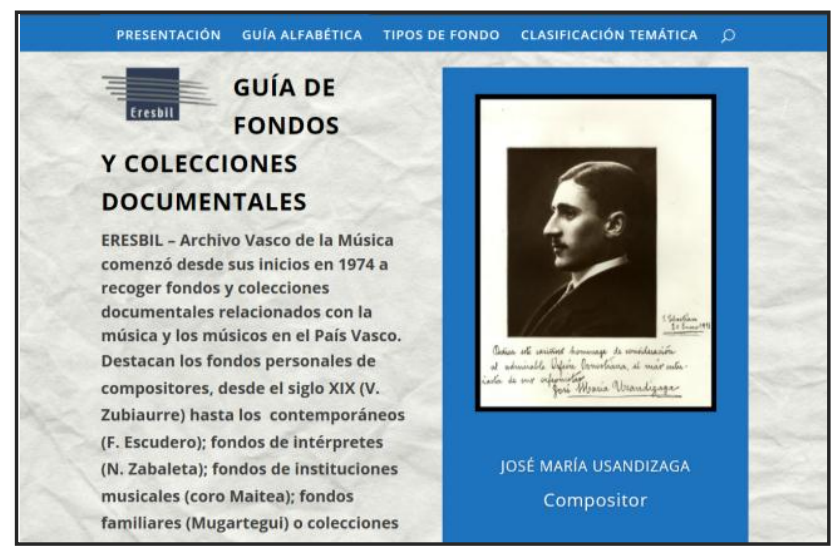

Fig. 2.

\section{3. BIBLIOTECA-HEMEROTECA}

El sistema de clasificación utilizado para la biblioteca de libre acceso es el adoptado por el colectivo de bibliotecas musicales del País $\mathrm{Vasco}^{2}$. Esta es la contabilización de la colección de libros de que dispone ERESBIL, incluyendo los libros pertenecientes a fondos de archivo:

. Obras de referencia

. Fundamentos generales de la musicología

. Etnomusicología

. Vida musical - Comercio musical - Instituciones musicales

\footnotetext{
${ }^{2}$ Musikako liburuen, partituren eta soinu-grabazioen sailkapen sistematikoa = Clasificación sistemática de libros de música, partituras y grabaciones sonoras. Vitoria-Gasteiz: Servicio Central de Publicaciones del Gobierno Vasco, 2007.
} 
JON BAGÜÉS

. Pedagogía musical 586

. Instrumentos musicales y su historia 698

. Historia de la música 4.572

. Teoría de la música - Ejecución musical $\quad 762$

. Música y otras ciencias y artes - Varios 1.588

. Biblioteca de complemento 716

TOTAL $\quad 15.787$

La biblioteca contiene asimismo una sección de partituras que conforman lo que en el archivo denominamos sección Universal para diferenciarla de la sección de compositores vascos. Es una sección que ha crecido especialmente en el apartado de colecciones monumentales, pero que también tiene secciones importantes de música vocal, música para órgano o música para guitarra. Hay que destacar en esta sección la Biblioteca Coral que desarrolla ERESBIL en unión con la Confederación de Coros del País Vasco.

Este es un resumen del número de títulos de que dispone ERESBIL a diciembre de 2016:

. Música vocal

. Música coral

. Música para piano e instrumentos de su familia

. Música para órgano

- Música para acordeón

. Música para instrumentos de percusión

. Música para instrumentos de cuerda punteada

. Música para instrumentos de viento

. Música para instrumentos de cuerda

. Música de cámara

. Música para agrupaciones y partituras de estudio

. Ediciones completas y colecciones monumentales

TOTAL

El número de títulos de revista que conserva ERESBIL es de 1.291. Entre los mismos se encuentran las siguientes colecciones completas:

. El Mundo musical (Madrid, 1845)

. Gaceta Musical de Madrid (1855-1856)

. La Ilustración Musical (Barcelona, 1883-1884) 
ERESBIL-ARCHIVo VASCO DE LA Música (ERRENTERIA):

UNA INSTITUCIÓN PARA LA PRESERVACIÓN Y DIFUSIÓN DE LA MÚSICA

. Revista Musical (Bilbao, 1903-1913)

. L’Orgue et les Organistes (Paris, 1924-1926)

. Txistulari (1928- )

. Anuario Musical (Barcelona, 1946- )

. Interamerican Music Bulletin (1957-1973)

. Current Musicology (1965- )

. Dantzariak (1970- )

. Early Music (1973- )

. Revista de Musicología (Madrid, 1978-)

. Revista de Folklore (Valladolid-Urueña, 1980- )

. Recerca Musicològica (Barcelona, 1981- )

. Imago musicae (1984- )

. Nasarre (Zaragoza, 1985- )

. Cahiers d'Ethnomusicologie (Genève, 1988- )

. Goldberg (Pamplona, 1997-2008)

Puede consultarse toda la información referida a los impresos musicales en el Catálogo general online a través del programa de gestión Absys. Esta sección surte de información a la Base de datos de Bibliografía musical vasca que recoge el vaciado, con resumen de contenido, de libros y artículos relacionados con la música vasca, así como la producción de los musicógrafos vascos. Esta base es también consultable online.

\subsection{FonOtECA Y AUDIOVISUALES}

La recopilación de documentos audiovisuales comienza a tener una actividad más regular a partir de 1986. El objetivo de ERESBIL en relación a los soportes sonoros y audiovisuales es recopilar la producción realizada en el País Vasco, así como la realizada fuera del mismo por intérpretes o con obras de compositores vasco-navarros. Para ello ERESBIL recibe por convenio un ejemplar de la producción discográfica ingresada por Depósito Legal en la Comunidad Autónoma del País Vasco, completando la colección con compras y donaciones. A fecha de diciembre de 2016 este es un resumen de las unidades documentales audiovisuales actualmente catalogadas en ERESBIL:

- Música académica

. Música de tradiciones nacionales

. Jazz

. Pop-Rock

. Infantil
27.253

18.976

1.677 
JON BAGÜÉS

. Grabaciones no musicales

500

TOTAL $\quad 104.610$

Del anterior volumen, 3.646 documentos son inéditos, siendo el restante documentos editados.

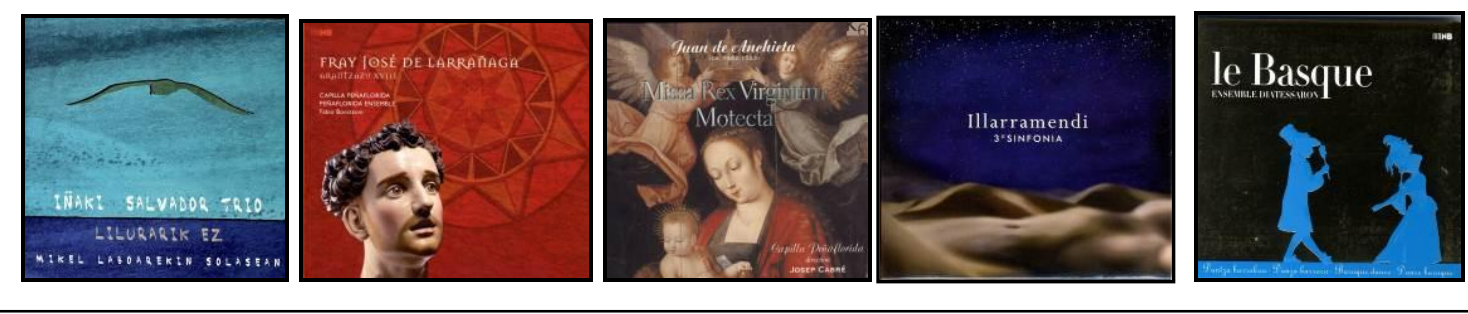

Fig. 3.

\subsection{DOCUMENTACIÓN}

Además de documentación de archivo, bibliográfica y fonográfica, ERESBIL recoge otros documentos que conforman la sección de documentación. Hay varios tipos de documentos que conforman importantes colecciones, como es el caso de las fotografías. ERESBIL tiene descritas en sus bases de datos más de 7.700 fotografías (incluyendo las pertenecientes a fondos de archivo). La documentación efímera compone otras dos importantes colecciones de ERESBIL. La colección propia de programas se trata a nivel de colección, constituyendo una ficha la suma de programas pertenecientes a una misma serie, alcanzando la cantidad de 1.289 fichas (Balerdi, 2000). Igual ocurre con la colección de carteles, que están descritos en 1.101 fichas.

Además de las citadas colecciones, prácticamente todos los compositores e intérpretes del País Vasco y Navarra tienen un dossier documental con información de su trayectoria musical, recogiendo artículos, recortes de prensa y documentos.

\section{CATÁLOgOS, gUías E INVENTARIOS}

Se puede acceder a ERESBIL y a sus contenidos de manera libre. La elaboración de los habituales instrumentos de descripción documental ha ido evolucionando desde el papel hasta las actuales posibilidades telemáticas. La documentación que hemos detallado previamente es tratada en ERESBIL atendiendo a su diversidad y en relación a los niveles documentales, recibiendo por lo tanto un tratamiento archivístico, un tratamiento bibliotecario o un tratamiento de información documental. 


\section{ERESBIL-ARCHIVo VASCO DE LA MúSICA (ERRENTERIA):}

\section{UNA INSTITUCIÓN PARA LA PRESERVACIÓN Y DIFUSIÓN DE LA MÚSICA}

Hemos señalado ya la Guía de fondos de archivo (Bagüés, 2002 y 2007), que ha sido realizada siguiendo la normativa ISAD $(G)$ de archivos, incorporando paulatinamente a la ficha a nivel de fondo el inventario correspondiente ${ }^{3}$.

La documentación impresa publicada recibe un tratamiento siguiendo el formato bibliotecario MARC ${ }^{4}$ ERESBIL ha realizado a lo largo de su trayectoria varios catálogos impresos específicos de sus fondos (Bagüés, 2002 y 2007; Landaberea y Bagüés, 1993, 2000a y 2000b; Leiñena y Bagüés, 2000).

La documentación tiene su tratamiento utilizando el programa FileMaker compartido con los fondos de archivo. Se catalogan así, a diferentes niveles, las fotografías, los programas, los carteles o los dosieres.

Recientemente se editó, a modo de prueba, el catálogo de obras de Francisco Escudero, como propuesta a desarrollar para reunir la documentación depositada en ERESBIL con la documentación pertinente ubicada en otras instituciones (Larrinaga, 2013).

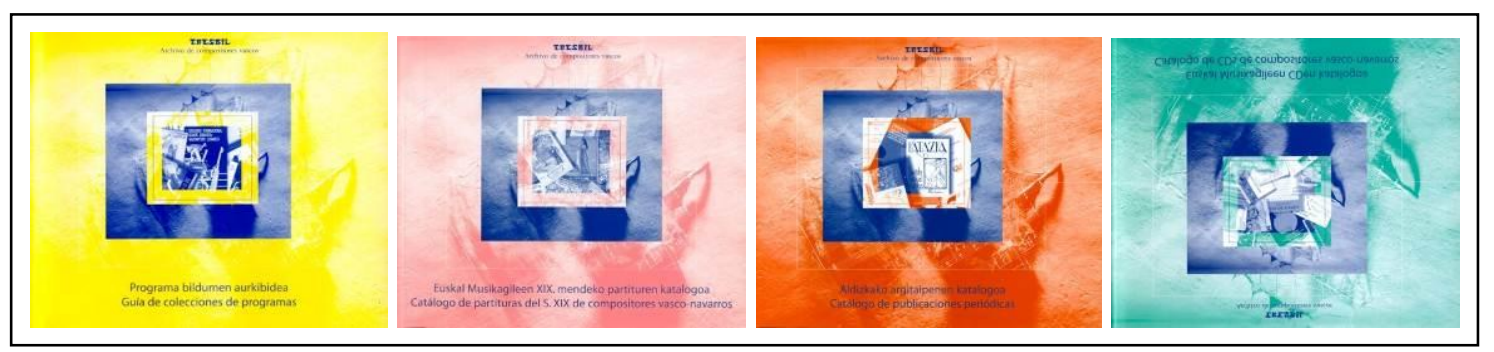

Fig. 4.

\section{ACTIVIDADES DE DIFUSIÓN DE ERESBIL}

ERESBIL diversifica igualmente sus distintas actividades en función de su finalidad de recuperación del patrimonio musical, de formación profesional, de apoyo a la investigación y a la publicación de estudios o de cooperación con las actividades de otras instituciones musicales del País Vasco.

\footnotetext{
${ }^{3}$ Se utiliza el programa informático Filemaker de manera que permite simultanear la descripción a nivel de fondo con la descripción a nivel de ítem. ERESBIL, por otra parte, colabora con el Archivo Histórico de Euskadi en la preservación de fondos de archivo.

${ }^{4}$ ERESBIL usa el programa de catalogación ABSYS, ofreciendo los datos al público mediante el programa AbsysNET. Incorpora asimismo periódicamente sus datos a BILGUNEA, catálogo colectivo integrado por diversas instituciones tanto públicas como privadas de la Comunidad Autónoma de Euskadi.
} 
JON BAGÜÉS

\subsection{MUSIKASTE}

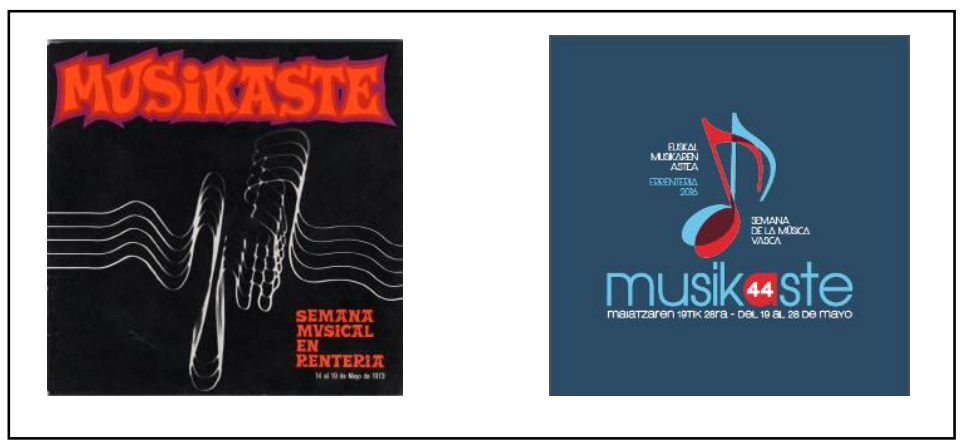

Fig. 5.

La evolución de la semana MUSIKASTE desde su primera edición el año 1973 en relación a su co-organización por parte de ERESBIL ha dado como resultado el que en la actualidad se haya convertido en la principal acción de difusión del archivo. José Luis Ansorena, director de la Coral Andra Mari y del archivo ERESBIL significaba la vinculación organizativa. A partir de su jubilación tanto como director artístico de la Coral y como director del archivo (2000), es ERESBIL quien asume su organización, incorporando la financiación a sus presupuestos, manteniendo la colaboración con la Coral Andra Mari en los aspectos prácticos.

ERESBIL difunde la obra de compositores vascos a través de la Semana MUSIKASTE, que se celebra anualmente en el mes de mayo.

En las 44 ediciones del festival celebradas hasta 2016 han sido interpretadas 2.428 composiciones escritas por 456 compositores de todos los tiempos, siendo 365 de estas obras estrenos absolutos. En el marco de los festivales se han presentado asimismo sesenta y tres ponencias sobre temas de música vasca y han tenido lugar nueve mesas redondas sobre problemática de la música vasca ${ }^{5}$.

\subsection{OTROS CONCIERTOS}

ERESBIL organizó a partir de 1980 en la primera sede del archivo el ciclo de conciertos ERESBIL-ERESIAK, repasando la evolución de las obras creadas por los compositores vascos al piano primero y al género de canto y piano después. Entre 2002 y 2009 se realizó una segunda serie de conciertos combinando la voz con la música de cámara.

\footnotetext{
${ }^{5}$ Con las ponencias presentadas en Musikaste 1978, con ocasión del primer centenario del fallecimiento de Hilarión Eslava, el equipo Musikaste-Eresbil coordinó la Monografía de Hilarión Eslava. La actividad desarrollada por el festival Musikaste durante sus primeros veinticinco años tiene su reflejo en la publicación de Ansorena (1997).
} 


\section{ERESBIL-ARChIVo VASCo De LA Música (ERRENTERIA): UNA INSTITUCIÓN PARA LA PRESERVACIÓN Y DIFUSIÓN DE LA MÚSICA}

De manera especial, y con motivo del 25 aniversario de la creación del archivo se organizaron varios conciertos estrenándose las obras ganadoras del Premio a la Creación Musical Contemporánea (1998).

\subsection{PUbliCACIONES IMPRESAS}

ERESBIL ha sido consciente desde su creación de la débil presencia de la impresión musical en el País Vasco, constituyendo un importante hándicap en la difusión de la obra de los compositores.

Promovió en un principio la edición facsimilar con el patrocinio de la Sociedad de Estudios Vascos, ofreciendo partituras de Dámaso Zabalza, Felipe Gorriti, José Ma Alvira y José de Uruñuela ${ }^{6}$, así como la colaboración en los dos cuadernos de música antigua con obras de Manuel Gamarra y Joaquín Ojinaga ${ }^{7}$.

En 1993 se comenzó a colaborar con la editorial CM-Ediciones Musicales, de Bilbao, publicando siete partituras de Norberto Almandoz como "Colección ERESBIL Bilduma" . En 2005 se publicaron cuatro partituras de Jesús García Leoz", y se continuó en 2006 con la colección de partituras "Euskal Musikagileak", dedicada principalmente a la edición de cuartetos de cuerda ${ }^{10}$. Como proyecto especial, financiado por la Diputación Foral de Gipuzkoa, se editaron entre 2004 y 2006 trece partituras, en su mayor parte sinfónicas, de Francisco Escudero en la editorial Tritó de Barcelona ${ }^{11}$. También ha colaborado ERESBIL en la edición del Cuarteto $\mathrm{n}^{\circ} 2$ de Jesús Guridi por Unión Musical Ediciones (2016).

La última difusión de partituras de compositores vascos ha sido el proyecto "Ez dok amairu hitzez hitz" compartido por la Cátedra Mikel Laboa de la Universidad del País

\footnotetext{
612 Estudios de mecanismo, 1er año, de Dámaso Zabalza (1982); 12 Estudios de mecanismo, 1er año, de Dámaso Zabalza (1982); Zortzileo, de Felipe Gorriti (1982); Romanza sin palabras, por José Ma Alvira (1982); El clavecín de Bendaña: vieja música vasca para piano, de José de Uruñuela (1982); Eusko Dantzak II, de José de Uruñuela (1984).

7 Juego de versos y sonatas, de Manuel de Gamarra. San Sebastián: Eusko Ikaskuntza, 1986; Obras musicales de Joaquin Ojinaga: recogidas y publicadas por José López-Calo. San Sebastián: Eusko Ikaskuntza, 1989.

${ }^{8}$ Urrundik: coro a 6 voces; Nere maitia, lo: coro a 6 voces; Gora Astigarraga!: Himno; Bocetos intermedios: piano; Berceuse: piano; Cuatro melodías de Iparraguirre: Canto y piano; Pequeña elegía para violoncello y piano.

9 Sonata para Violin y Piano; Primer cuarteto en Fa\# menor, Cuarteto con Piano; Llanto a Manolete: para cuerda y guitarra.

${ }_{10}$ Cuarteto en Sol n 1, de Jesús Guridi (2006); Cuarteto modal, de Tomás Aragüés Bernad (2007); Cuarteto en Mi, de Aita Donostia (2009); Lied (para cuarteto de cuerda), de Aita Donostia (2009); Quatuor à cordes sur des thèmes populaires basques, de José $\mathrm{M}^{\mathrm{a}}$ Usandizaga (2012).

${ }_{11}$ Hojas de Album, para orquesta de cuerda (2004); Gnosis: para quinteto de viento (2004); Uranzu: para acordeón (2004); Concierto para clavecín y sexteto orquestal (2004); Fantasía geosinfónica: para orquesta de acordeones, percusión, txirula, txalaparta y barítono (2004); Sinfonía no 5 "Ultreia" (2005); Illeta: Oratorio elegíaco para barítono, coro y orquesta (2005); Poème symphonique, op. 3: para orquesta (2006); Nocturno: para orquesta (2006); El sueño de un bailarin: Ballet para orquesta (2006); Evocación en Iciar - Preludio matinal: para orquesta (2006); Preludio: para orquesta (2006); Zigor. Ópera en cuatro actos (2006). Todas las anteriores partituras aparecen englobadas en la serie "Euskal Musikagileak", figurando ERESBIL en el pie de imprenta. Diversas ayudas institucionales del Gobierno Vasco han incluido en la misma serie de la editorial Tritó las obras Leyenda vasca y Sinfonía Pirenaica, de Jesús Guridi, editadas ambas el año 2007.
} 
JON BAGÜÉS

Vasco y la Capitalidad Cultural de San Sebastián 2016, editando doce adaptaciones corales de otras tantas melodías de la nueva canción vasca ${ }^{12}$.

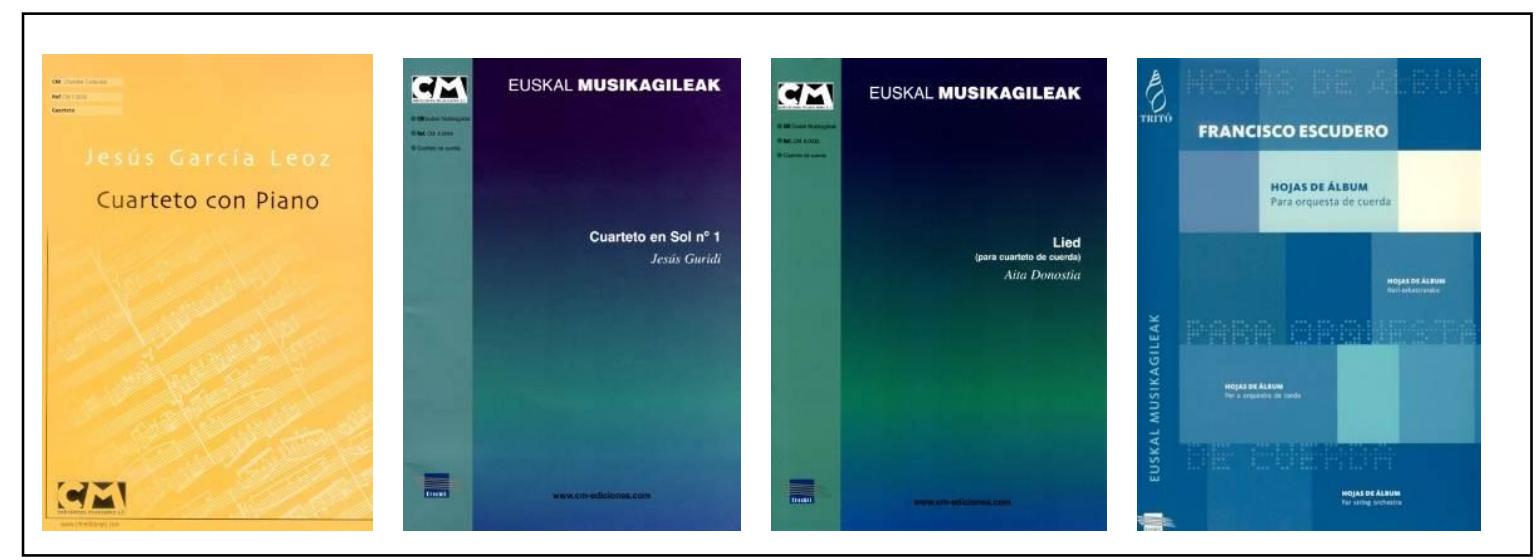

Fig. 6 .

\subsection{PUbLiCACIONES ONLINE}

Es en el año 2003 cuando ERESBIL publica su primera página web ${ }^{13}$. En ella se puede consultar el catálogo de obras de compositores vasco-navarros, el catálogo general de libros, publicaciones periódicas, grabaciones sonoras y partituras, así como la base de datos de vaciado bibliográfico y el directorio de Recursos Musicales del País Vasco.

ERESBIL comenzó a incorporar paulatinamente documentos digitales al portal digital vasco LIBURUKLIK, donde de momento pueden consultarse partituras y grabaciones sonoras.

El año 2008 ERESBIL amplía la oferta digital con la realización de páginas web temáticas y de personalidades musicales, pudiendo consultarse las siguientes:

\section{. PÁGINAS WEB TEMÁTICAS \\ . Editoriales Musicales del País Vasco y Navarra \\ . Los comienzos de la industria discográfica en Euskal Herria \\ . Casas y Sellos Discográficos del País Vasco y Navarra \\ . Colección de cilindros de cera. Fondo Familia Ybarra}

\footnotetext{
12 Egunsentia [texto y música de Benito Lertxundi; adaptación de Jesús Egiguren]; Sehaska kanta (Amaren bularra) [texto y música de Xabier Lete; adaptación de David Azurza]; Euskalerri nerea [texto y música de Xabier Lete; adaptación de Junkal Guerrero]; Eq, eq dut nabi [texto y música de Julen Lekuona; adaptación de Idoia Azurmendi]; Ez gaude konforme [texto y música de Lourdes Iriondo; adaptación de Eva Ugalde]; Ez kanta beltra [texto y música de Benito Lertxundi; adaptación de Francisco Ibáñez]; Gure bide galduak [texto y música de Benito Lertxundi; adaptación de Xabier Sarasola]; Itsasoan urak haundi dire [texto y música de Julen Lekuona; adaptación de Javier Busto]; Lore gorrien balada [texto y música de Xabier Lete; adaptación de Koldo Uriarte]; Poeta horiek [texto y música de Xabier Lete; adaptación de Josu Elberdin]; Umeek ere jakin dute [texto de Anjel Lertxundi; música de Benito Lertxundi; adaptación de Iker González]; Urak dakarren [texto y música de Jose Angel Irigarai; adaptación de Koldo Pastor]. Las partituras serán accesibles a través del espacio sitio digital de Eresbil en Liburuklik.

13 Puede consultarse el aspecto que tenía en 2008 en http://www.ondarenet.kultura.ejgv.euskadi.eus:8085/wayback/es/*/http://www.eresbil.com
} 
ERESBIL-ARCHIVO VASCO DE LA MÚSICA (ERRENTERIA):

UNA INSTITUCIÓN PARA LA PRESERVACIÓN Y DIFUSIÓN DE LA MÚSICA

- Catálogos discográficos ${ }^{14}$

. El Txistu. Fuentes, documentos sonoros, repertorios en Eresbil

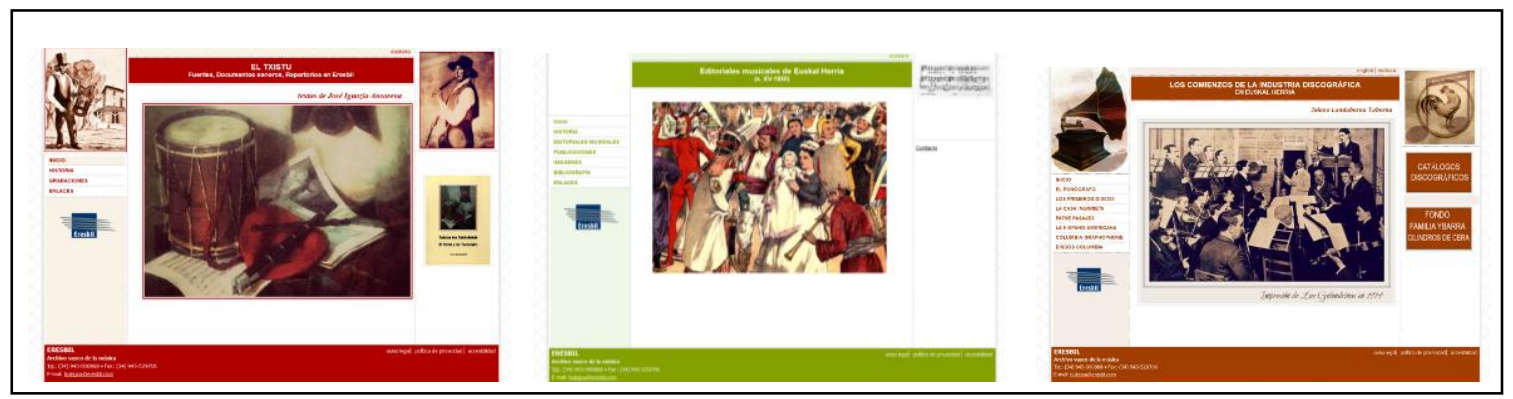

Fig. 7.

. PÁGINAS WEB DE PERSONALIDADES MUSICALES

. Luis de Aramburu (1905-1999)

. José Antonio Arana Martija (1931-2011)

. Francisco Escudero (1912-2002)

. Jesús Guridi (1886-1961)

. Raimundo Sarriegui (1838-1913)

. Sara Soto (1941-1999)

. Losé Ma Usandizaga (1887-1915)

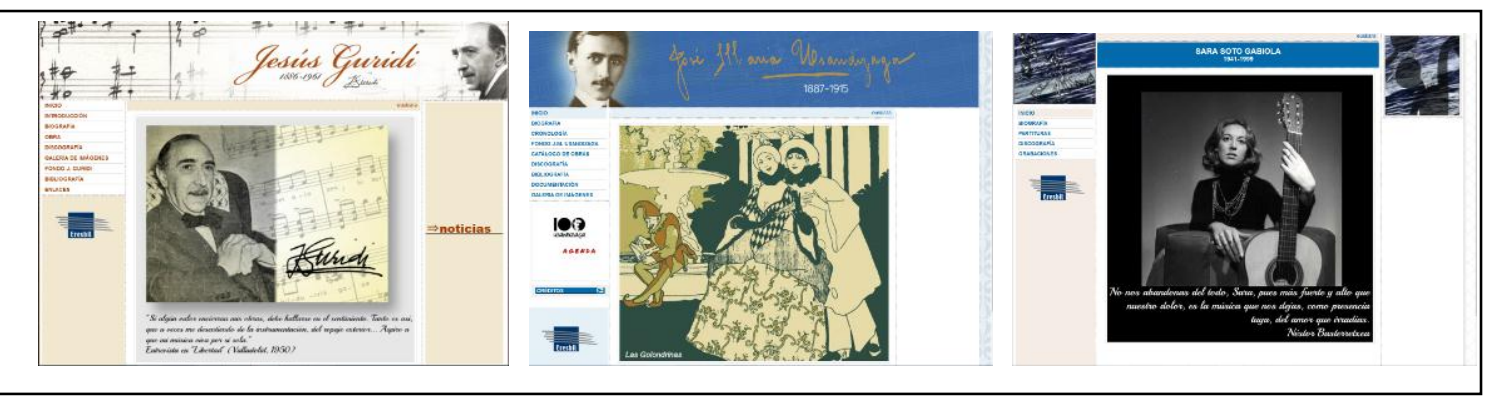

Fig. 8.

${ }^{14}$ Se trata, como se explica más adelante, del proyecto colaborativo "Matriz" realizada en el seno de AEDOM, Asociación Española de Documentación Musical y coordinado por ERESBIL, un catálogo colectivo de catálogos impresos de sellos fonográficos editados entre 1903 y 1954, muy útil para la datación de registros sonoros antiguos. Puede consultarse el proyecto en http://www.aedom.org/matriz. 
JON BAGÜÉS

\section{COOPERACIÓN E INVESTIGACIÓN DESDE ERESBIL}

ERESBIL participa activamente en la Asociación Internacional de Bibliotecas, Archivos y Centros de Documentación Musicales (AIBM) ${ }^{15}$, así como en la rama española AEDOM y pertenece asimismo a la Asociación Internacional de Archivos Sonoros (IASA) $^{16}$. Es la sede de Musikagileak, Asociación Vasco-Navarra de Compositores.

Especial actividad ha desplegado el archivo en el seno de la AIBM, implicándose sobre todo en el proyecto AMA-Access to Music Archives de censo y descripción de fondos de archivo y colecciones musicales. Coordina en el ámbito de España dicho censo en colaboración con otras instituciones en el marco de la Asociación Española de

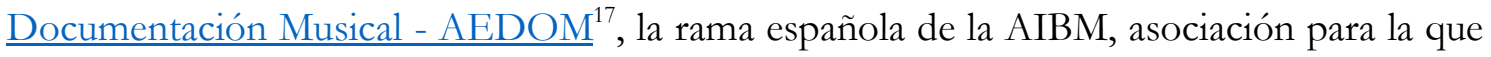
ha coordinado asimismo el proyecto $\underline{\text { Matriz, }}$, repertorio colectivo de catálogos discográficos existentes en las instituciones españolas, realmente útil para la datación de grabaciones antiguas.

Además de las entidades citadas, ERESBIL coopera de manera estrecha con entidades y colectivos específicos con el propósito de promocionar y desarrollar:

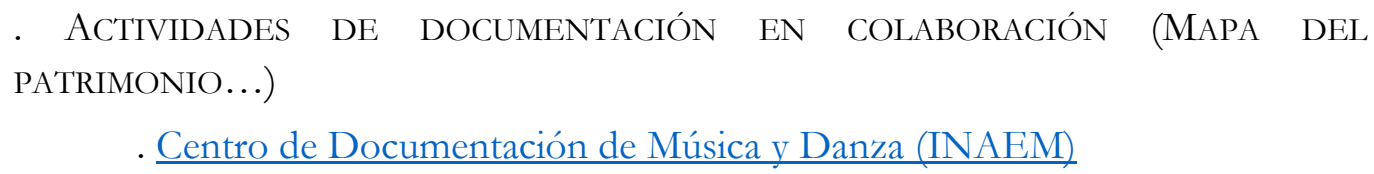

- Asociación Vasca de Profesionales de Archivos, Bibliotecas y Centros de Documentación (ALDEE)

- ACTIVIDADES DE RELACIONES CON MEDIOS MUSICALES

. Asociación Vasco-Navarra de Compositores $^{18}$

. Asociación de Txistularis del País Vasco ${ }^{19}$

. Confederación de Coros del País Vasco ${ }^{20}$

. Euskal Herriko Trikitixa Elkartea ${ }^{21}$

\section{. ACTIVIDADES RELACIONADAS CON ENTIDADES INTERPRETATIVAS}

. Orquesta Sinfónica de Euskadi2 22

. Orfeón Donostiarra ${ }^{23}$

\footnotetext{
15 http://www.iaml.info/

16 http://www.iasa-web.org/

17 http://www.aedom.org/

18 http://musikagileak.com/es/

19 http://www.txistulari.com/

20 http://koralakeae.com/

21 http://trikitixa.eus/

22 http://www.euskadikoorkestra.es/es/default.asp

23 http://www.orfeondonostiarra.org/es/. Con ocasión de la celebración del centenario de la fundación del Orfeón Donostiarra, Eresbil editó en 1997 la Discografía del Orfeón Donostiarra: 1897-1997, en realización, Jaione Landaberea.
} 
ERESBIL-ARCHIVO VASCO DE LA MÚSICA (ERRENTERIA):

UNA INSTITUCIÓN PARA LA PRESERVACIÓN Y DIFUSIÓN DE LA MÚSICA

. ACTIVIDADES RELACIONADAS CON EDITORIALES Y PRODUCTORAS

. Elkar Argitaletxea

. $\underline{\text { Badok }}$

\section{. ACTIVIDADES RELACIONADAS CON ENTIDADES DIFUSORAS}

. Institut Culturel Basque $^{24}$

\section{. Cursos de Verano de la Universidad del País VAsco}

Un aspecto fundamental entre las finalidades de ERESBIL es el de la recuperación documental juntamente con el de la investigación relacionada con la música. Se han llevado a cabo proyectos concretos de recopilación patrimonial en colaboración con Eusko Ikaskuntza-Sociedad de Estudios Vascos, en concreto en la localidad navarra de Lesaka. Igualmente colaboró con la asociación de proyectos educativos Ikertze en la recopilación de canciones de cuna.

Juntamente con MUSIKENE, Centro Superior de Música del País Vasco ${ }^{25}$, participó en el proyecto "Garat", de recuperación del repertorio para cuarteto de cuerda, proyecto que implicaba la catalogación, promoción e interpretación de cuartetos de cuerda de compositores vascos. Esta relación estrecha con MUSIKENE se está consolidando en la realización de proyectos compartidos de difusión e investigación patrimonial.

ERESBIL ha formado parte en los dos Cursos de Doctorado en Musicología en colaboración con la EHU-UPV impartidos los años 2004-2006. Asimismo, mantiene relación con la Universidad del País Vasco colaborando con la Cátedra Mikel Laboa en varios proyectos, especialmente en los contenidos de las páginas web impulsadas por la Cátedra ${ }^{26}$.

ERESBIL ha convocado entre 2004 y 2011, en colaboración con Euskal Fundazioa, la Juan Zelaia Letamendi Musika Beka, destinada a trabajos de investigación que aúnen la música con el euskera, logrando 4 trabajos que pueden consultarse online.

ERESBIL colabora como EPO en el proyecto I+D+i HAR2011-24295 Música de cámara instrumental y vocal en España en los siglos XIX y XX: recuperación, recepción, análisis crítico y estudio comparativo del género en el contexto europeo, coordinado en la Universidad de Granada.

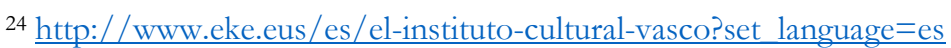

25 http://musikene.eus/musikene/

26 Página dedicada a Mikel Laboa: http://www.ehu.eus/mikellaboa/home. Están en proceso de realización las páginas dedicadas a los cantautores Xabier Lete, Lourdes Iriondo e Imanol.
} 
JON BAGÜÉS

\section{UN NUEVo RETO DE ERESBIL: LA DOCUMENTACión DE DANZA EN EL PAÍ́s VASCo}

En el año 2009 y ante la ausencia de un centro documental especializado en danza en los territorios de la Comunidad Autónoma Vasca, la Consejería de Cultura del Gobierno Vasco propuso a ERESBIL el inicio de una colección documental relacionada con la danza en el País Vasco.

La ubicación dentro de nuestro archivo se propuso inicialmente como temporal, pudiendo ser trasladada en el futuro a la biblioteca de un hipotético centro dedicado a la danza. Mientras, ERESBIL se ha comprometido a ir conformando una colección documental lo más completa e interesante posible, para que sea consultada tanto por los profesionales y aficionados a la danza como por la ciudadanía en general.

La biblioteca consta ya de 983 monografías y 539 documentos audiovisuales, entre los que destacan las videograbaciones relacionadas con los proyectos de promoción de danza contemporánea del Gobierno Vasco así como de la Diputación Foral de Gipuzkoa. Toda la información puede consultarse online agrupada bajo la colección Dantza, dividida en los videos de danza contemporánea (173) y los videos de danza tradicional (366), proyecto este último de recogida, análisis y descripción detallada de contenido de videos inéditos de danza tradicional vasca, promovido por el Gobierno Vasco.

Finalizamos esta presentación del archivo ERESBIL y sus actividades con una escueta alusión al entorno digital como un desafío documental y de difusión del presente y futuro cercano. La documentación musical del presente se elabora en gran medida de manera digital, y uno de los principales retos de los sistemas e instituciones documentales es ya el grado de profundidad, extensión y fiabilidad en la preservación del patrimonio que generan las actuales generaciones de creadores, intérpretes, promotores e investigadores. Sin lugar a dudas, la colaboración y complementariedad entre las instituciones será una de las claves del éxito.

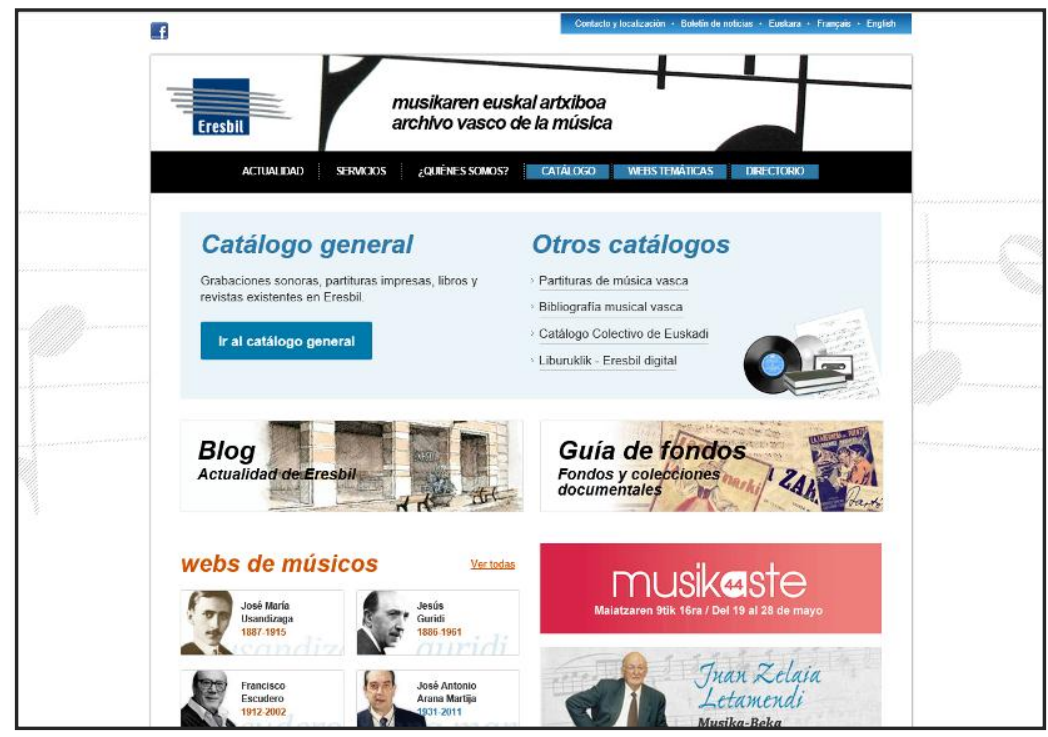

Fig. 9. 


\section{ERESBIL-ARCHIVO VASCO DE LA MÚSICA (ERRENTERIA):}

UNA INSTITUCIÓN PARA LA PRESERVACIÓN Y DIFUSIÓN DE LA MÚSICA

\section{BIBLIOGRAFÍA}

Ansorena, J. L. (1997). Musikaste, 25 urte $=25$ años de Musikaste. San Sebastián: Kutxa Fundazioa.

Balerdi, B. y Bagüés (coords.) (2000). Eresbil-Archivo de Compositores Vascos: Programa bildumen aurkibidea $=$ Guia de colecciones de programas. Errenteria: Eresbil.

Bagüés, Jon (2000). “Archivos musicales en Eresbil-Archivo Vasco de la Música”. Musiker, XIII, pp. 221-252.

Bagüés, Jon (2007). "Fondos musicales en bibliotecas y archivos de Euskal Herria". Musiker, XV, pp. 271-295.

ERESBIL (coord.) (1978). Monografía de Hilarión Eslava. Pamplona: Institución Príncipe de Viana.

Landaberea, J. y Bagüés, J. (coords.) (1993). Eresbil-Archivo de Compositores Vascos: Catálogo de Publicaciones periódicas. Donostia: Asociación de Bibliotecarios Documentalistas de Gipuzkoa.

Landaberea, J. y Bagüés, J. (2000a). Eresbil- Archivo de Compositores Vascos: Aldizkako Argitalpemnen Katalogoa $=$ Catálogo de Publicaciones periódicas. Errenteria: Eresbil.

Landaberea, J. y Bagüés, J. (2000b). Eresbil-Archivo de Compositores Vascos: Euskal musikagileen CDen katalogoa $=$ Catálogo de CDs de compositores vasco-navarros. Errenteria: Eresbil.

Leiñena, P. y Bagüés, J. (2000). Eresbil-Archivo de Compositores Vascos: Euskal Musikagileen XIX. Mendeko Partituren Katalogoa $=$ Catálogo de Partituras del s. XIX de compositores vasconavarros. Errenteria: Eresbil.

Larrinaga, Itziar (2013). Francisco Escudero. Catálogo razonado de obras. Errenteria: Eresbil.

Fecha de recepción: 18/11/2016

Fecha de aceptación: 14/12/2016 